\title{
LETTERE AL DIRETTORE
}

ANGELO PISTOIA

Istituto di Matematica Generale e Finanziaria

Università degli Studi di Pavia

pervenuta il 10 settembre 1980

Ti sarò grato se nel prossimo numero della RIVISTA DI MATEMATICA PER LE SCIENZE ECONOMICHE E SOCIALI verrà inserita la seguente

\section{ERRATA}

A. Pistora, About internal rate of return, Atti del III Convegno A.M.A.S.E.S., Napoli 29-31 Ottobre 1979, Dottrinari Editore Salerno.

pag. riga in luogo di

$2916+\quad$ Pisa

$2948+\quad$ A.M.A.S.E.S. Milano leggasi

Pavia

Fascicoli dell'Istituto di Mat. Gen. e Fin., Università di Pavia, N. 59. 